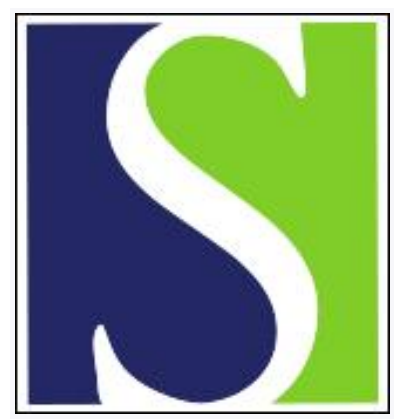

Scand J Work Environ Health 2021;47(8):600-608

https://doi.org/10.5271/sjweh.3989

Published online: 26 Sep 2021, Issue date: 01 Nov 2021

Sickness absence and return to work among employees with knee osteoarthritis with and without total knee arthroplasty: a prospective register linkage study among Finnish public sector employees

by Kaila-Kangas L, Leino-Arjas P, Koskinen A, Takala E-P, Oksanen T, Ervasti J, Kausto J

It is not known whether conservative treatment of knee osteoarthritis $(\mathrm{OA})$ is associated with shorter or longer return to work as compared with total knee arthroplasty (TKA). We examined the duration of sickness absence due to $O A$ and return to work among public sector employees. We differentiated between employees with and without TKA.

Affiliation: Finnish Institute of Occupational Health, Box 18, 00032 TYÖTERVEYSLAITOS, Finland. leena.kaila-kangas@ttl.fi

Key terms: arthritis; cohort study; employment; Finland; Finland; knee arthroplasty; knee osteoarthritis; musculoskeletal disease; prospective; public sector employee; register; return to work; sick leave; sickness absence; work

This article in PubMed: www.ncbi.nlm.nih.gov/pubmed/34564731 


\title{
Sickness absence and return to work among employees with knee osteoarthritis with and without total knee arthroplasty: a prospective register linkage study among Finnish public sector employees
}

\author{
by Leena Kaila-Kangas, PhD, ${ }^{1}$ Päivi Leino-Arjas, MD, ${ }^{1}$ Aki Koskinen, MSc, ${ }^{1}$ Esa-Pekka Takala, MD, ${ }^{1}$ Tuula Oksanen, MD, ${ }^{2}$ Jenni \\ Ervasti, PhD, ${ }^{1}$ Johanna Kausto, PhD ${ }^{1}$
}

\begin{abstract}
Kaila-Kangas L, Leino-Arjas P, Koskinen A, Takala E-P, Oksanen T, Ervasti J, Kausto J. Sickness absence and return to work among employees with knee osteoarthritis with and without total knee arthroplasty: a prospective register linkage study among Finnish public sector employees. Scand J Work Environ Health. 2021;47(8):600-608. doi:10.5271/sjweh.3989
\end{abstract}

\begin{abstract}
Objective This study aimed to examine duration of sickness absence due to knee osteoarthritis (OA) and sustained return to work (RTW) among municipal employees who had at least one compensated sickness absence period due to knee OA. The contribution of sociodemographic characteristics, diabetes and previous sickness absence were assessed. We differentiated between participants with and without total knee arthroplasty (TKA).

Methods Data from 123506 employees in the Finnish Public Sector Study were linked with national health and mortality register information. There were 3231 sickness absence periods ( 2372 participants) due to knee OA in 2005-2011. Kaplan-Meier curves for sustained RTW were obtained and median time with inter-quartile range (IQR) calculated for those with and without TKA. Cox regression analyses were carried out in multivariable analyses.
\end{abstract}

Results The median time to RTW from the beginning of sickness absence was 21-28 days when TKA was not related to sickness absence and 92-145 days when it was. Among participants with no TKA, age 60-64, nonsedentary work, diabetes, and previous sickness absences predicted longer time to RTW, while pain medication predicted a shorter time. Among participants with TKA, non-sedentary work and previous sickness absences predicted a longer time to RTW.

Conclusions The clinical relevance of the difference in time to RTW between employees with or without TKA was substantial. Employees with knee OA working in physically demanding jobs need work modifications after TKA, and this calls for a dialog between occupational health care professionals and workplaces.

Key terms arthritis, cohort study, employment, Finland; musculoskeletal disease, sick leave, work.

Knee osteoarthritis $(\mathrm{OA})$ is a common chronic disorder that causes pain and functional limitations with difficulties in walking, stair climbing and other lower-extremity tasks. It reduces quality of life (1), work ability, and work participation $(2,3)$. The etiology of knee OA is not fully understood, but genetic factors, older age, female sex, obesity, injuries, and repetitive loading of the knee joint, eg, frequent squatting, kneeling, and heavy lifting may play a role $(4,5)$. OA is a complex condition that involves the entire joint, with progressive loss of articular cartilage, synovitis, and changes in the subchondral bone (6). There is often a discrepancy between the sever- ity of symptoms and the severity of knee OA as assessed by radiography, related to individual experience of pain, and the discrepancy can be to either direction (7).

According to a systematic review (8), the prevalence estimates of knee OA have varied considerably depending on the definition. In European studies, the self-reported prevalence has varied between $7.1 \%$ and $15.0 \%$. Among Finns aged $\geq 30$ years, the prevalence of clinically assessed knee OA was $6 \%$ and $8 \%$ among men and women respectively in 2000 (9). The incidence of knee $\mathrm{OA}$ is higher among women than men, and it increases with age and increasing relative weight of

1 Finnish Institute of Occupational Health, Helsinki, Finland.

2 Institute of Public Health and Clinical Nutrition, University of Eastern Finland, Kuopio, Finland.

Correspondence to: Leena Kaila-Kangas, Specialized Researcher, Finnish Institute of Occupational Health, Box 18, 00032 TYÖTERVEYSLAITOS, Finland. [E-mail: leena.kaila-kangas@ttl.fi] 
the population (8). Thus, the occurrence of knee OA is likely to rise due to population aging and the obesity epidemic (10).

The management of knee OA is primarily conservative emphasizing the role of exercise, balance training and weight control (7) in diminishing pain and improving functional capacity $(11,12)$. Arthroscopic surgery with debridement or partial meniscectomy may not bring long-lasting benefits in pain or physical function $(13,14)$. In severe cases, total knee arthroplasty (TKA) has been regarded as the best method to reduce pain and disability and maintain physical activity in the long term $(15,16)$.

According to a review (17), there is limited evidence for a specific symptom threshold that justifies TKA, but the minimum requirements for it are significant, prolonged symptoms with supporting clinical and radiological signs. Obesity, diabetes and smoking, and comorbidities particularly among older patients increase the risk of complications following TKA. On average $15-20 \%$ of patients are dissatisfied with their outcome after surgery, usually because of ongoing pain and poor physical functioning (18). Also, low preoperative functioning, comorbidities (diabetes mellitus, pulmonary disease and back pain), psychological distress, or desire to return to high-impact activities and unrealistic expectations have been associated with dissatisfaction following TKA (19).

Most employees return to work after TKA (20-22), but long periods of sickness absence are common (23), and only a minority of patients aged $\geq 60$ years ever return to work (24). A systematic review (25) identified several individual-related factors that were associated with return to work (RTW) after TKA. These included sex, age, educational level, obesity, motivation to return, and physical and mental functioning, as well as workrelated factors like physical demands, workplace characteristics, employment status, pre-operative sickness absence and workers' compensation.

Timely RTW is the primary goal after recovery among working-aged TKA patients, and the recent steep increase in the incidence of TKA $(18,26)$ has raised concern regarding the consequences of the procedure for RTW and employability (27). In several countries, the current number of TKA patients $<65$ years is already high, and the figures are expected substantially to rise at least in United States and Europe (27). It is suggested that also the number of revised TKA is rising in those countries (28). According to a meta-analysis (29), the expectable revision rate in ten years has been $<5 \%$ in general, but $7 \%$ among patients aged $<60$ years.

Ability to work is not defined by only health or health impairment, but also in relation to one's work demands, and the onset of illness or handicap may not necessarily lead to work disability (30). Because of a lack of research, it is not known whether conservative treatment of knee OA is associated with a shorter or longer time to RTW as compared with TKA. Therefore, we examined time to sustained RTW among Finnish municipal sector employees with sickness absence due to knee OA, using comprehensive register data. We stratified the analyses by treatment modality (TKA or no TKA) and assessed the contribution of morbidity related factors, sociodemographic characteristics, and occupation.

\section{Methods}

\section{Participants}

The study cohort consisted of participants of the Finnish Public Sector study working in ten municipalities and six hospital districts representing about $20 \%$ of public sector employees in Finland (31). In the current analyses, we focused on those employees who were working in the target organizations at the start of the follow-up on 1 January $2005(\mathrm{~N}=123$ 506) and had at least one compensated (ie, lasting $>10$ work days) sickness absence period due to knee OA before the end of follow-up on 31 December 2011. There were 169330 compensated sickness absence periods (from 65985 participants) due to any disorder during the follow-up. Of these periods, $3231(1.9 \%)$ were due to knee OA (among 2372 participants). Data on hospitalization due to TKA in 1996-2004 were explored, and those who had undergone TKA before the study period were excluded from the analyses.

\section{Data sources}

Employers' registers provided information on sociodemographic factors of the participants. Data on medically certified sickness absence periods were drawn from the national register of the Social Insurance Institution of Finland. The register covers the compensated periods that lasted for $>10$ workdays including the day when the disability started. Information on hospitalization periods and the surgical procedures targeting knee $\mathrm{OA}$, also on TKA, (recorded according to the Nordic Classification of Surgical Procedures) was received from the Hospital Discharge Register provided by the National Institute for Health and Welfare. Data on reimbursed medication purchases with codes from the Anatomical Therapeutic Chemical (ATC) classification system were retrieved from the Register of Special Reimbursement for Medication Purchases from the Social Insurance Institution of Finland. We obtained data on pensions from the national registers of the Finnish Centre for Pensions, and data on 
deaths were retrieved from Statistics Finland. Register data were combined by each participant's personal identification number that is assigned to each citizen of Finland at birth or when receiving a residence permit.

The Ethics Committee of the Hospital District of Helsinki and Uusimaa in Finland approved the study protocol.

\section{The Finnish work disability compensation scheme}

In Finland, all non-retired residents aged 16-67 are eligible for a compensation of incapacity to work due to disorders after the employer period of payment (a minimum of ten days), and thereafter the Social Insurance Institution of Finland compensates sickness absence $>10$ days. The maximum length of sickness absence compensation is 300 working days per disease in two years. In case of longer-term work disability, a disability pension can be granted either temporarily or permanently.

\section{Sustained return to work (RTW)}

RTW was defined as sustained when it was indicated by the end of sickness absence period that was not followed by a recurrent sickness absence period due to the same diagnosis within 30 days. This was based on the regulations of the Social Insurance Institution of Finland, according to which two consecutive sickness absence periods with the same diagnosis are regarded as one if the working period in between the two absence periods lasted $<30$ calendar days.

Sickness absence data consisted of the starting and ending dates and diagnostic codes (ICD-10) (32) for all medically certified and compensated sickness absence periods. For the analysis, the duration of each sickness absence period was calculated starting from the initial day of work absence until the end of the compensation period. Sickness absence periods that lasted $\leq 10$ days, and thus were not compensated, were not available in the data. We did not differentiate between partial and full sickness absence days in the analyses.

\section{Covariates}

Covariates were sex, age at the time of sickness absence, and occupation [categorized according to the International Classification of Occupations (ISCO) as follows: managers and professionals (ISCO 1-2), associate professionals and clerks (ISCO 3-4), service and care workers (ISCO 5) and manual workers (ISCO 6-9)]. Region was categorized according to the geographical location of the participant's work organization, to adjust for possible regional differences in sickness absence practices (data not shown). Health-related covariates were (i) sickness absence lasting $>10$ days during the previous year due to musculoskeletal disorders (no/ yes), and separately due to other disorders (no/yes), respectively (ii), purchased medication for musculoskeletal pain (no/yes) using ATC classification system codes M01 (anti-inflammatory and anti-rheumatic products) and N02 (analgesics) (iii), diabetes (based on entitlement to special reimbursements (no/yes), and (iv) hospitalization periods related to knee OA. Medication purchases and hospitalization periods were recorded for the preceding 30 days and during the following 7 days after the sickness absence period. To adjust for the severity of knee OA related to sickness absence periods with no TKA, we added information on hospitalizations (no/yes) due to knee OA.

\section{Statistical analyses}

The observational unit was a medically certified and compensated sickness absence period ( $>10$ workdays) due to knee OA. We analyzed separately those periods that were associated with TKA and those that were not. The participants could belong only to one of these categories. We excluded sickness absence associated with partial TKA (36 periods).

The Cox proportional hazards model with a cluster option was applied to estimate the time from the beginning of the sickness absence period to RTW. The cluster option considered the intra-individual correlation of sickness absence periods by using the robust sandwich variance estimates in calculating standard errors. In case of hospitalization for TKA, the time to RTW was estimated from the beginning of sickness absence period due to knee OA or from the beginning of the hospitalization period (when there was no previous sickness absence from work due to knee OA).

Hazard ratios (HR) and 95\% confidence intervals (CI) were calculated for the time from the beginning of sickness absence to RTW. Observations were right censored in case of disability pension, old age pension, death, or end of the follow-up, whichever came first. Cox proportional hazards models were adjusted for age, sex, occupational group, region, sickness absence during previous year for musculoskeletal disorders, and for other disorders, reimbursed purchase of pain medication, and diabetes. The time until RTW was visually presented by Kaplan Maier curves, separately for those who had undergone TKA and for those who had not. All analyses were performed using SAS software package (version 9.4; SAS Institute, Inc, Cary, NC, USA). 


\section{Results}

In this sample of municipal workers, knee OA-related sickness absence periods ( $\mathrm{N}=3231$ ) comprised $1.9 \%$ of all compensated ( $>10$ days) sickness absence periods $(\mathrm{N}=169330)$ and $6.2 \%$ of sickness absence periods due to musculoskeletal disorders ( $\mathrm{N}=51$ 469) between 2005 and 2011. Of the participants with sickness absence due to knee OA, $17.6 \%(\mathrm{~N}=416)$ had undergone TKA.

\section{Characteristics of participants with sickness absence due to knee osteoarthritis (OA)}

Among participants with sickness absence due to OA, the proportion of women was $77.4 \%$, and among those with TKA, $80.1 \%$ (table 1). The mean age at the time of the first sickness absence due to knee OA among those with no TKA ( $\mathrm{N}=1956)$, was 52.8 years (SD 7.2) and with TKA 56.5 years (SD 5.2) at the time of surgery. Seventy-one per cent of those with TKA belonged to the age group of 55 to 64 at the time of surgery. The proportion of participants aged $60-64$ was $19.5 \%$ among those without TKA and $31.0 \%$ among those with TKA. The corresponding figures among manual workers were $33.2 \%$ and $21.9 \%$.

\section{Return to work (RTW)}

Table 2 presents the adjusted associations between baseline characteristics and RTW when sickness absence due to knee OA was not related to TKA. The median time until RTW was 21-28 days. Sex or hospitalization related to sickness absence due to knee OA were not associated with RTW. Participants aged 60-64 had a lower probability of RTW compared to those aged 26-49 (HR 0.78, 95\% CI 0.68-0.88). With managers and professionals as reference, associate professionals and clerks $(0.85,95 \%$ CI $0.75-0.97)$, service and care workers (HR $0.76,95 \%$ CI $0.67-0.86$ ) and manual workers (HR $0.74,95 \%$ CI $0.65-0.85$ ) had a lower probability of RTW. Similarly, sickness absence due to musculoskeletal disorders overall (HR $0.90,95 \% \mathrm{CI}$ $0.83-0.98$ ) or due to other disorders (HR $0.87,95 \% \mathrm{CI}$ $0.79-0.95)$ during the previous year, as well as diabetes (HR $0.65,95 \%$ CI $0.53-0.80$ ), decreased the probability of RTW, while purchase of pain medication (yes versus no) shortened it (HR 1.09, 95\% CI 1.01-1.18).

Table 3 presents the adjusted associations between baseline characteristics and RTW when sickness absence was related to TKA. The median time until RTW was 92-145 days. Sex had no association, but occupation was strongly associated with time to RTW: while the median time to RTW was 92 days among managers and professionals, the corresponding figures were 108 among associate professionals and clerks (HR 0.51, 95\% CI $0.38-0.68$ with managers and professionals as reference), 130 among service and care workers (HR 0.34, 95\% CI $0.26-0.46$ ), and 145 among manual workers (HR 0.28, 95\% CI 0.20-0.39). The occurrence of sickness absence due to musculoskeletal disorders (HR 0.79, 95\% CI $0.64-0.97$ ) or other disorders (HR $0.71,95 \% \mathrm{CI}$ $0.53-0.94$ ) during the previous year was associated with a longer time to RTW.

Kaplan-Meier curves (figure 1) demonstrate that $92 \%$ of the compensated sickness absence periods due to knee OA without TKA ended with RTW within six months, while the corresponding figure among those with TKA was $72 \%$. Within a year, $96 \%$ and $89 \%$ of the periods, respectively, had ended with RTW.

Table 1. Characteristics of participants. [OA=0steoarthritis]

\begin{tabular}{|c|c|c|c|c|c|c|}
\hline & \multicolumn{2}{|c|}{$\begin{array}{l}\text { All patients } \\
(\mathrm{N}=2372)\end{array}$} & \multicolumn{2}{|c|}{$\begin{array}{l}\text { Knee OA patients with no knee } \\
\text { arthroplasty }(\mathrm{N}=1956)\end{array}$} & \multicolumn{2}{|c|}{$\begin{array}{l}\text { Knee OA patients with knee } \\
\text { arthroplasty }(\mathrm{N}=416)\end{array}$} \\
\hline & $\mathrm{N}$ & $\%$ & $\mathrm{~N}$ & $\%$ & $\mathrm{~N}$ & $\%$ \\
\hline \multicolumn{7}{|l|}{ Sex } \\
\hline Men & 536 & 22.6 & 457 & 23.4 & 79 & 19.0 \\
\hline Women & 1836 & 77.4 & 1499 & 76.6 & 337 & 80.1 \\
\hline \multicolumn{7}{|l|}{ Age (years) } \\
\hline $26-39$ & 116 & 4.9 & 114 & 5.8 & 2 & 0.5 \\
\hline $40-44$ & 189 & 8.0 & 179 & 9.1 & 10 & 2.4 \\
\hline $45-49$ & 327 & 13.8 & 293 & 15.0 & 34 & 8.2 \\
\hline $50-54$ & 517 & 21.8 & 442 & 22.6 & 75 & 18.0 \\
\hline $55-59$ & 713 & 30.1 & 547 & 28.0 & 166 & 39.9 \\
\hline $60-64$ & 510 & 21.5 & 381 & 19.5 & 129 & 31.0 \\
\hline \multicolumn{7}{|l|}{ Occupational group } \\
\hline Managers and professionals & 371 & 15.6 & 292 & 14.9 & 79 & 19.0 \\
\hline Associate professionals and clerks & 586 & 24.7 & 464 & 23.7 & 122 & 29.3 \\
\hline Service and care workers & 674 & 28.4 & 550 & 28.1 & 124 & 29.8 \\
\hline Manual workers & 741 & 31.2 & 650 & 33.2 & 91 & 21.9 \\
\hline
\end{tabular}


Table 2. Determinants and time to sustained return to work (RTW) among knee osteoarthritis (OA) patients without knee arthroplasty ( $N=1956)$. Fully a adjusted Cox proportional hazards regression model with a cluster option. Hazard ratios (HR) and 95\% confidence intervals (CI).

\begin{tabular}{|c|c|c|c|c|c|c|}
\hline \multirow[t]{2}{*}{ Baseline characteristics } & \multicolumn{3}{|c|}{ Sickness absence periods ( $N=2776$ ) } & \multicolumn{3}{|c|}{ Days until RTW ${ }^{\mathrm{b}}$ (percentiles) } \\
\hline & $\mathrm{N}$ & $\mathrm{HR}$ & $95 \% \mathrm{Cl}$ & $25^{\text {th }}$ & $50^{\text {th }}$ & $75^{\text {th }}$ \\
\hline \multicolumn{7}{|l|}{ Sex } \\
\hline Men & 651 & Ref & & 15 & 21 & 35 \\
\hline Women & 2125 & 1.05 & $0.95-1.16$ & 15 & 21 & 34 \\
\hline \multicolumn{7}{|l|}{ Age (years) } \\
\hline $26-49 c$ & 79 & Ref & & 15 & 21 & 35 \\
\hline $50-54$ & 666 & 1.03 & $0.92-1.15$ & 15 & 21 & 34 \\
\hline $55-59$ & 830 & 0.91 & $0.81-1.01$ & 16 & 23 & 40 \\
\hline $60-64$ & 481 & 0.74 & $0.65-0.84$ & 17 & 26 & 50 \\
\hline \multicolumn{7}{|l|}{ Occupational group } \\
\hline Managers and professionals & 358 & Ref & & 15 & 21 & 35 \\
\hline Associate professionals and clerks & 630 & 0.84 & $0.73-0.96$ & 16 & 24 & 43 \\
\hline Service and care workers & 816 & 0.75 & $0.65-0.86$ & 17 & 25 & 49 \\
\hline Manual workers & 972 & 0.74 & $0.64-0.84$ & 17 & 26 & 50 \\
\hline \multicolumn{7}{|c|}{$\begin{array}{l}\text { Sickness absence during previous year for musculoskeletal } \\
\text { disorders }\end{array}$} \\
\hline No & 1708 & Ref & & 15 & 21 & 35 \\
\hline Yes & 1068 & 0.90 & $0.83-0.98$ & 16 & 23 & 40 \\
\hline \multicolumn{7}{|c|}{$\begin{array}{l}\text { Sickness absence during previous year for other than muscu- } \\
\text { loskeletal disorders }\end{array}$} \\
\hline No & 2196 & Ref & & 15 & 21 & 35 \\
\hline Yes & 580 & 0.85 & $0.77-0.94$ & 16 & 24 & 42 \\
\hline \multicolumn{7}{|c|}{ Hospitalization related to sickness absence due to knee $\mathrm{OA}$} \\
\hline No & 1975 & Red & & 15 & 21 & 35 \\
\hline Yes & 801 & 1.02 & $0.93-1.12$ & 15 & 21 & 34 \\
\hline \multicolumn{7}{|c|}{ Reimbursed purchase of pain medication } \\
\hline No & 1214 & Ref & & 15 & 21 & 35 \\
\hline Yes & 1562 & 1.12 & $1.03-1.22$ & 14 & 20 & 32 \\
\hline \multicolumn{7}{|l|}{ Comorbidity: diabetes } \\
\hline No & 2650 & Ref & & 15 & 21 & 35 \\
\hline Yes & 126 & 0.65 & $0.53-0.80$ & 17 & 28 & 61 \\
\hline
\end{tabular}

a Region was also adjusted for.

${ }^{\mathrm{b}}$ Estimated time to RTW when in the reference category of other covariates.

'26-year-old was the youngest.

Table 3. Determinants and time to sustained return to work (RTW) among knee osteoarthritis (OA) patients with total knee arthroplasty (N=416). Fully a adjusted Cox proportional hazards regression model with a cluster option. Hazard ratios (HR) and 95\% confidence intervals (Cl).

\begin{tabular}{|c|c|c|c|c|c|c|}
\hline \multirow[t]{2}{*}{ Baseline characteristics } & \multicolumn{3}{|c|}{ Sickness absence periods ( $N=455)$} & \multicolumn{3}{|c|}{ Days until RTW ${ }^{\mathrm{b}}$ (percentiles) } \\
\hline & $\mathrm{N}$ & HR & $95 \% \mathrm{Cl}$ & 25 th & 50 th & 75th \\
\hline \multicolumn{7}{|l|}{ Gender } \\
\hline Men & 83 & Ref & & 87 & 92 & 108 \\
\hline Women & 372 & 1.00 & $0.75-1.32$ & 87 & 92 & 108 \\
\hline \multicolumn{7}{|l|}{ Age (years) } \\
\hline $36-49^{c}$ & 50 & Ref & & 87 & 92 & 108 \\
\hline $50-54$ & 80 & 0.87 & $0.60-1.28$ & 89 & 94 & 115 \\
\hline $55-59$ & 180 & 1.18 & $0.84-1.66$ & 84 & 92 & 100 \\
\hline $60-64$ & 141 & 1.21 & $0.85-1.72$ & 84 & 92 & 99 \\
\hline \multicolumn{7}{|l|}{ Occupational group } \\
\hline Managers and professionals & 87 & Ref & & 87 & 92 & 108 \\
\hline Associate professionals and clerks & 135 & 0.51 & $0.38-0.68$ & 92 & 108 & 154 \\
\hline Service and care workers & 134 & 0.34 & $0.26-0.46$ & 94 & 130 & 245 \\
\hline Manual workers & 99 & 0.28 & $0.20-0.39$ & 96 & 145 & 325 \\
\hline \multicolumn{7}{|c|}{$\begin{array}{l}\text { Sickness absence during previous year for musculoskeletal } \\
\text { disorders }\end{array}$} \\
\hline No & 280 & Ref & & 87 & 92 & 108 \\
\hline Yes & 175 & 0.79 & $0.64-0.97$ & 89 & 95 & 121 \\
\hline \multicolumn{7}{|c|}{$\begin{array}{l}\text { Sickness absence during previous year for other than musculo- } \\
\text { skeletal disorders }\end{array}$} \\
\hline No & 374 & Ref & & 87 & 92 & 108 \\
\hline Yes & 81 & 0.69 & $0.52-0.92$ & 90 & 96 & 129 \\
\hline \multicolumn{7}{|c|}{ Reimbursed purchase of pain medication } \\
\hline No & 177 & Ref & & 87 & 92 & 108 \\
\hline Yes & 278 & 1.07 & $0.86-1.34$ & 86 & 92 & 104 \\
\hline \multicolumn{7}{|l|}{ Comorbidity: diabetes } \\
\hline No & 436 & Ref & & 87 & 92 & 108 \\
\hline Yes & 19 & 0.85 & $0.63-1.14$ & 89 & 94 & 117 \\
\hline
\end{tabular}

a Region was also adjusted for.

${ }^{\mathrm{b}}$ Estimated time to RTW when in the reference category of other covariates.

b36-year old was the youngest. 


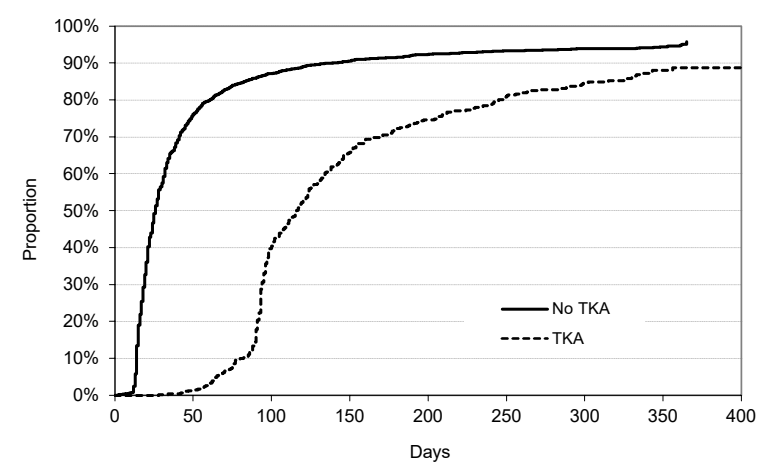

Figure 1. Cumulative proportion of employees with return to workfrom baseline to 365 days for sickness absence episodes due to knee osteoarthritis with and without total knee arthroplasty (TKA).

\section{Discussion}

In this register based, prospective study, we analyzed the time to RTW from the beginning of sickness absence due to knee OA in a cohort of municipal employees who had at least one compensated sickness absence period due to knee OA during the 7-year follow-up. Sustained RTW was defined as the end of the sickness benefit period that was not followed by recurrent sickness absence period for the same diagnosis within 30 days (33). It was assumed that a person was recovered from an acute disorder when she/he had been working 30 days after sickness absence.

Analyses were carried out separately for sickness absence periods (and participants) not related to TKA and for those thus related. At the time of the first sickness absence due to knee OA not related to TKA, the participants were on average 4 years younger than those with TKA, whose mean age was 56 years at the time of surgery.

The median time until sustained RTW was 21-28 and 92-145 days among those without and those with TKA, respectively, when adjusting for sociodemographic characteristics, diabetes and previous sickness absence. Among participants with no TKA, the age of 60-64 years, non-sedentary work, diabetes, and previous sickness absence due to musculoskeletal or other disorders, predicted longer time to RTW, while pain medication predicted a shorter time. Among those with TKA, non-sedentary work and previous sickness absence due to musculoskeletal or other disorders predicted longer time to RTW. A prolonged sickness absence was especially common in service, care and manual work.

The primary strength of our study was the prospective study design and access to comprehensive and valid national register data on sickness absence and hospital- ization (34). We could also adjust for many important factors that may have an impact on the time until RTW, including health conditions based on register data on all causes of sickness absence and reimbursed purchases of medication. A cohort of mostly female municipal employees representing hundreds of occupations was followed up. Although municipal occupations involve a large variety of tasks, the results may not be directly generalizable to other branches of industry. Public sector employees are mostly women. Even though the proportion of women was much higher than that of men, the number of men was high enough for comparison. Moreover, the previous studies have shown contradictory sex related results on the time to RTW among TKA patients $(20,35)$.

There are some limitations in this study. First, we did not have information on short sickness absences. Consequently, the length of sickness absence until RTW may be slightly overestimated. The sickness absence periods related with knee OA comprised $1.9 \%$ of all sickness absence periods in our data, in line with a Swedish population-based study that assessed that $2 \%$ of all sick days were attributable to knee OA (36). Another limitation is a lack of information on body weight of the participants. There is evidence on obese patients having more difficulties with knee OA and less improvement in TKA outcomes (37). However, diabetes was associated with slower RTW only among sickness absence not related to TKA. This result may be due to healthrelated selection of individuals to TKA, while diabetes increases the risk of complications following TKA (17). Selection of older patients to surgery or postponement of it due to work-related hindrances can also explain the overrepresentation of those aged 60-64 and the underrepresentation of manual workers among TKA patients.

Most of the previous studies on time to RTW after TKA have based on self-reported retrospective data, and the authors have calculated the full or partial return to work based on the working hours before and after TKA (35). We considered only full return to work. We also measured the time to RTW from the first day of sickness absence and this may have resulted in a longer median time to RTW than in previous studies. In a register-based study, about half of the patients started their sick leave even before the surgery (23).

Lastly, there may have been changes in the care path for OA in Finland after 2005-2011 when the data were gathered. For example, a new law, called the '30-60-90-day rule', was introduced in 2012. This law requires that, after 30 days of sickness absence, the employer needs to inform their occupational healthcare services about the situation of the employee. After 60 days of sickness absence, the occupational healthcare physician needs to assess the need for rehabilitation. And after 90 days of sickness absence, the occupational 
healthcare physician needs to assess the possibilities of RTW. A register-based study (38) of municipal workers showed, that the effect of the new legislation was most pronounced after about 12 weeks of sickness absence, but the annual gain in work participation was modest.

There is a lack of research on RTW when knee OA is not related to TKA. In a study (39) among Swedish working-age population, the length of the age- and sexadjusted mean period of sickness absence for knee OA was 81 days. A population-based register study on work ability and work participation of Finnish employees with knee OA (40) found that the median time to RTW was 31 days. However, neither of these studies reported whether sickness absence was related with TKA or not. In a previous study that was based on the same data as the study at hand, the median time to RTW for intervertebral disc disorders (ICD-10: M51) was 32 days among men and 37 among women, and for back pain (ICD-10: M54) 21 and 22 days, respectively (33).

In this study, $92 \%$ of the participants with knee OA who did not go through TKA returned to work within six months and about $96 \%$ within a year. Half of the sickness absence periods ended with RTW in 21 days. Thus, the median time to RTW was comparable to sickness absence related to back pain in the same population (33). When sickness absence was related to TKA return to work took several months.

According to a descriptive review, the greatest functional improvements occur during the first year after the TKA (41). We found that $72 \%$ of sickness absence periods compensated for TKA were associated with RTW within six months, and $89 \%$ in one year. The adjusted median time to RTW was 3.1 months among managers and professionals, 3.6 months among associate professionals and clerks, 4.3 months among service and care workers, and 4.8 months in manual work. A systematic review (20) of studies (altogether 649 patients) on RTW after TKA showed that $71-83 \%$ of patients were back at work within 3-6 months. In the study of Sankar et al (22), 77\% returned to work in 6 months after TKA. Early return to work (within one month) was associated with male sex, university education, working in business, finance or administration, and low physical demands at work. In a prospective study of working patients aged $<65$ years undergoing TKA for OA, 89\% had returned to work one year postoperatively (42). In line with our results, a recent review found that most important factors associated with a slower return to work were a physical nature of employment and preoperative absence from work (35).

We used occupational group as a proxy for physical workload. However, the loads imposed on the knee can vary even within the same job due to individual variation of tasks. It was found (43) that the risk of sickness absence and disability pension due to knee OA was higher in female-dominated job sectors such as health care, childcare and cleaning, which are common among municipal occupations. A large Finnish nationally representative study suggested that $50 \%$ of disability retirement due to knee OA among individuals in most manual occupations could be attributed to physical workload factors (44).

A qualitative study (45) reported, that the rehabilitation goals of TKA patients are mainly focused on knee mobility and range of motion, but there is also a need to integrate work into the individually tailored rehabilitation process. However, this requires a dialog between healthcare professionals and employers on appropriate work adjustments (46). Especially occupational health care professionals have knowledge on the work, workplace and potential work adjustments. Obviously, more knowledge is needed on how rehabilitation combined with workplace adjustments can ease RTW and possibly postpone TKA among knee OA patients.

To conclude, our findings showed that $50 \%$ of employees on sickness absence due to knee OA returned to work in three weeks when TKA was not needed, and in three months when it was. RTW was especially slow in non-sedentary jobs, and among those with previous sickness absences. Given the burden of knee OA in working age population, the clinical relevance of the difference in time to RTW between employees with or without TKA was substantial, and especially among manual workers. Employees with knee OA working in physically demanding jobs need work modifications after TKA, and this calls for a dialog between occupational healthcare professionals and workplaces.

\section{Acknowledgement}

The Finnish Work Environment Fund partly funded this study (grant number 115371). The authors declare no conflict of interest.

\section{References}

1. Vitaloni M, Botto-van Bemden A, Sciortino Contreras RM, Scotton D, Bibas M, Quintero M et al. Global management of patients with knee osteoarthritis begins with quality of life assessment: a systematic review. BMC Musculoskelet Disord 2019 Oct;20(1):493. https://doi.org/10.1186/s12891019-2895-3.

2. Palmer KT, Milne P, Poole J, Cooper C, Coggon D. Employment characteristics and job loss in patients awaiting surgery on the hip or knee. Occup Environ Med 2005 Jan;62(1):54-7. https://doi.org/10.1136/oem.2004.014977. 
3. Sayre EC, Li LC, Kopec JA, Esdaile JM, Bar S, Cibere J. The effect of disease site (knee, hip, hand, foot, lower back or neck) on employment reduction due to osteoarthritis. PLoS One 2010 May;5(5):e10470. https://doi.org/10.1371/ journal.pone.0010470.

4. Visser AW, de Mutsert R, le Cessie S, den Heijer M, Rosendaal FR, Kloppenburg M; NEO Study Group. The relative contribution of mechanical stress and systemic processes in different types of osteoarthritis: the NEO study. Ann Rheum Dis 2015 Oct; 74(10):1842-7. https://doi. org/10.1136/annrheumdis-2013-205012.

5. Verbeek J, Mischke C, Robinson R, Ijaz S, Kuijer P, Kievit A et al. Occupational exposure to knee loading and the risk of osteoarthritis of the knee: a systematic review and a dose-response meta-analysis. Saf Health Work 2017 Jun;8(2):130-42. https://doi.org/10.1016/j. shaw.2017.02.001.

6. Glyn-Jones S, Palmer AJ, Agricola R, Price AJ, Vincent $\mathrm{TL}$, Weinans $\mathrm{H}$ et al. Osteoarthritis. Lancet 2015 Jul;386(9991):376-87. https://doi.org/10.1016/S01406736(14)60802-3.

7. Sharma L. Osteoarthritis of the Knee. N Engl J Med 2021 Jan;384(1):51-9. https://doi.org/10.1056/NEJMcp1903768.

8. Pereira D, Peleteiro B, Araújo J, Branco J, Santos RA, Ramos E. The effect of osteoarthritis definition on prevalence and incidence estimates: a systematic review. Osteoarthritis Cartilage 2011 Nov;19(11):1270-85. https:/ doi.org/10.1016/j.joca.2011.08.009.

9. Heliövaara M, Impivaara O, Nykyri E. Hilkka Riihimäki. Changes in morbidity. In Kaila-Kangas L, ed. Musculoskeletal disorders and diseases in Finland. Results of the Health 2000 Survey. Publications of the National Public Health Institute, B25/2007: 60-69. Available from: http:// www.julkari.fi/bitstream/handle/10024/78197/2007b25.pdf.

10. Zhang Y, Jordan JM. Epidemiology of osteoarthritis. Clin Geriatr Med 2010 Aug;26(3):355-69. https://doi. org/10.1016/j.cger.2010.03.001.

11. Fernandes L, Hagen KB, Bijlsma JW, Andreassen O, Christensen P, Conaghan PG et al.; European League Against Rheumatism (EULAR). EULAR recommendations for the non-pharmacological core management of hip and knee osteoarthritis. Ann Rheum Dis 2013 Jul;72(7):112535. https://doi.org/10.1136/annrheumdis-2012-202745.

12. Bannuru RR, Osani MC, Vaysbrot EE, Arden NK, Bennell K, Bierma-Zeinstra SM et al. OARSI guidelines for the non-surgical management of knee, hip, and polyarticular osteoarthritis. Osteoarthritis Cartilage 2019 Nov;27(11):1578-89. https://doi.org/10.1016/j. joca.2019.06.011.

13. Thorlund JB, Juhl CB, Roos EM, Lohmander LS. Arthroscopic surgery for degenerative knee: systematic review and meta-analysis of benefits and harms. BMJ 2015 Jun;350:h2747. https://doi.org/10.1136/bmj.h2747.

14. Brignardello-Petersen R, Guyatt GH, Buchbinder R, Poolman RW, Schandelmaier S, Chang Y et al. Knee arthroscopy versus conservative management in patients with degenerative knee disease: a systematic review. BMJ Open 2017 May;7(5):e016114. https://doi.org/10.1136/ bmjopen-2017-016114.

15. Meding JB, Meding LK, Ritter MA, Keating EM. Pain relief and functional improvement remain 20 years after knee arthroplasty. Clin Orthop Relat Res 2012 Jan;470(1):144-9. https://doi.org/10.1007/s11999-011-2123-4.

16. Shan L, Shan B, Suzuki A, Nouh F, Saxena A. Intermediate and long-term quality of life after total knee replacement: a systematic review and meta-analysis. J Bone Joint Surg Am 2015; 21;97(2):156-68.

17. Adie S, Harris I, Chuan A, Lewis P, Naylor JM. Selecting and optimising patients for total knee arthroplasty. Med J Aust 2019 Feb;210(3):135-41. https://doi.org/10.5694/ mja2.12109.

18. Price AJ, Alvand A, Troelsen A, Katz JN, Hooper G, Gray A et al. Knee replacement. Lancet 2018 Nov;392(10158):1672-82. https://doi.org/10.1016/S01406736(18)32344-4.

19. Husain A, Lee GC. Establishing realistic patient expectations following total knee arthroplasty. J Am Acad Orthop Surg 2015 Dec;23(12):707-13. https://doi. org/10.5435/JAAOS-D-14-00049.

20. Tilbury C, Schaasberg W, Plevier JW, Fiocco M, Nelissen RG, Vliet Vlieland TP. Return to work after total hip and knee arthroplasty: a systematic review. Rheumatology (Oxford) 2014 Mar;53(3):512-25. https://doi.org/10.1093/ rheumatology/ket389.

21. Lombardi AV Jr, Nunley RM, Berend KR, Ruh EL, Clohisy JC, Hamilton WG et al. Do patients return to work after total knee arthroplasty? Clin Orthop Relat Res 2014 Jan;472(1):138-46. https://doi.org/10.1007/s11999-0133099-z.

22. Sankar A, Davis AM, Palaganas MP, Beaton DE, Badley EM, Gignac MA. Return to work and workplace activity limitations following total hip or knee replacement. Osteoarthritis Cartilage 2013 Oct;21(10):1485-93. https:// doi.org/10.1016/j.joca.2013.06.005.

23. Stigmar K, Dahlberg LE, Zhou C, Jacobson Lidgren H, Petersson IF, Englund M. Sick leave in Sweden before and after total joint replacement in hip and knee osteoarthritis patients. Acta Orthop 2017 Apr;88(2):152-7. https://doi.or g/10.1080/17453674.2016.1269051.

24. Scott CE, Turnbull GS, MacDonald D, Breusch SJ. Activity levels and return to work following total knee arthroplasty in patients under 65 years of age. Bone Joint J 2017 Aug;99-B(8):1037-46. https://doi.org/10.1302/0301620X.99B8.BJJ-2016-1364.R1.

25. Pahlplatz TM, Schafroth MU, Kuijer PP. Patient-related and work-related factors play an important role in return to work after total knee arthroplasty: a systematic review. Journal of ISAKOS: Joint Disorders \& Orthopaedic Sports Medicine 2017;2:127-32. https://doi.org/10.1136/ jisakos-2016-000088. 
26. Carr AJ, Robertsson O, Graves S, Price AJ, Arden NK, Judge A et al. Knee replacement. Lancet 2012 Apr;379(9823):1331-40. https://doi.org/10.1016/S01406736(11)60752-6.

27. Kuijer PP, Burdorf A. Prevention at work needed to curb the worldwide strong increase in knee replacement surgery for working-age osteoarthritis patients [Editorial]. Scand J Work Environ Health 2020 Sep;46(5):457-60. https://doi. org/10.5271/sjweh.3915.

28. Postler A, Lützner C, Beyer F, Tille E, Lützner J. Analysis of total knee arthroplasty revision causes. BMC Musculoskelet Disord 2018 Feb;19(1):55. https://doi.org/10.1186/s12891018-1977-y.

29. Lützner J, Hübel U, Kirschner S, Günther KP, Krummenauer F. Langzeitergebnisse in der Knieendoprothetik : Metaanalyse zu Revisionsrate und funktionellem Ergebnis [Long-term results in total knee arthroplasty. A metaanalysis of revision rates and functional outcome] [German.]. Chirurg $2011 \mathrm{Jul} ; 82(7): 618-24$. https://doi. org/10.1007/s00104-010-2001-8.

30. Mattila-Holappa P, Kausto J, Aalto V, Kaila-Kangas L, Kivimäki M, Oksanen T et al. Alternative duty work as workplace-initiated procedure to reduce sickness absence. BMC Public Health 2021 Jun;21(1):1154. https://doi. org/10.1186/s12889-021-11181-1.

31. Kivimäki M, Gimeno D, Ferrie JE, Batty GD, Oksanen T, Jokela $\mathrm{M}$ et al. Socioeconomic position, psychosocial work environment and cerebrovascular disease among women: the Finnish public sector study. Int J Epidemiol 2009 Oct;38(5):1265-71. https://doi.org/10.1093/ije/dyn373.

32. International Classification of Diseases and Related Health Problems (ICD-10), 10th Revision, version 2016. World Health Organisation (WHO). Available from: http://apps. who.int/classifications/icd10/browse/2016/en.

33. Kausto J, Pentti J, Oksanen T, Virta LJ, Virtanen M, Kivimäki $\mathrm{M}$ et al. Length of sickness absence and sustained return-to-work in mental disorders and musculoskeletal diseases: a cohort study of public sector employees. Scand J Work Environ Health 2017 Jul;43(4):358-66. https://doi. org/10.5271/sjweh.3643.

34. Sund R. Quality of the Finnish Hospital Discharge Register: a systematic review. Scand J Public Health 2012 Aug;40(6):505-15. Epub 2012 Aug 16. https://doi. org/10.1177/1403494812456637.

35. Van Leemput D, Neirynck J, Berger P, Vandenneucker H. Return to work after primary total knee arthroplasty under the age of 65 years: A systematic review. J Knee Surg 2021 Jan.

36. Hubertsson J, Petersson IF, Thorstensson CA, Englund M. Risk of sick leave and disability pension in working-age women and men with knee osteoarthritis. Ann Rheum Dis 2013 Mar;72(3):401-5. https://doi.org/10.1136/ annrheumdis-2012-201472.
37. McGrory BJ, Weber KL, Jevsevar DS, Sevarino K. Surgical Management of Osteoarthritis of the Knee: evidence-based Guideline. J Am Acad Orthop Surg 2016 Aug;24(8):e87-93. https://doi.org/10.5435/JAAOS-D-16-00159.

38. Halonen JI, Solovieva S, Pentti J, Kivimäki M, Vahtera J, Viikari-Juntura E. Effectiveness of legislative changes obligating notification of prolonged sickness absence and assessment of remaining work ability on return to work and work participation: a natural experiment in Finland. Occup Environ Med 2016 Jan;73(1):42-50. https://doi. org/10.1136/oemed-2015-103131.

39. Hubertsson $\mathrm{J}$, Englund $\mathrm{M}$, Hallgårde $\mathrm{U}$, Lidwall $\mathrm{U}$, Löfvendahl S, Petersson IF. Sick leave patterns in common musculoskeletal disorders--a study of doctor prescribed sick leave. BMC Musculoskelet Disord 2014 May;15:176. https://doi.org/10.1186/1471-2474-15-176.

40. Kontio T, Viikari-Juntura E, Solovieva S. Effect of Osteoarthritis on Work Participation and Loss of Working Life-years. J Rheumatol 2020 Apr;47(4):597-604. https:// doi.org/10.3899/jrheum.181284.

41. Canovas F, Dagneaux L. Quality of life after total knee arthroplasty. Orthop Traumatol Surg Res 2018 Feb;104 1S:S41-6. https://doi.org/10.1016/j.otsr.2017.04.017.

42. Leichtenberg CS, Tilbury C, Kuijer P, Verdegaal S, Wolterbeek R, Nelissen R et al. Determinants of return to work 12 months after total hip and knee arthroplasty. Ann R Coll Surg Engl 2016 Jul;98(6):387-95. Epub 2016 May 3. https://doi.org/10.1308/rcsann.2016.0158.

43. Hubertsson J, Turkiewicz A, Petersson IF, Englund M. Understanding Occupation, Sick Leave, and Disability Pension Due to Knee and Hip Osteoarthritis From a Sex Perspective. Arthritis Care Res (Hoboken) 2017 Feb;69(2):226-33. https://doi.org/10.1002/acr.22909.

44. Kontio T, Viikari-Juntura E, Solovieva S. To what extent do education and physical work load factors explain occupational differences in disability retirement due to knee OA? A nationwide register-based study in Finland. BMJ Open 2019 Feb;8(11):e023057. https://doi.org/10.1136/ bmjopen-2018-023057.

45. Bardgett M, Lally J, Malviya A, Deehan D. Return to work after knee replacement: a qualitative study of patient experiences. BMJ Open 2016 Feb;6(2):e007912. https://doi. org/10.1136/bmjopen-2015-007912.

46. Grant M, O-Beirne-Elliman J, Froud R, Underwood $\mathrm{M}$, Seers K. The work of return to work. Challenges of returning to work when you have chronic pain: a metaethnography. BMJ Open 2019 Jun;9(6):e025743. https://doi. org/10.1136/bmjopen-2018-025743.

Received for publication: 13 March 2021 